\title{
ANNOTATION
}

\section{The Cosmetic Eye}

An interesting paper on circumocular dermatitis was published by Ereaux, of Montreal, in the Canadian Medical Association journal of April, 1940. It had been read before the Section of Dermatology at the Annual Meeting of the Canadian Medical Association in June, 1939. Two types are described: 1, a widespread facial dermatitis in which the eyelids participate and 2, cases where only the eyelids are affected. A large number of cases suffer from seborrhoea, in which the greasy, scaly skin retains cosmetics and allows of their penetration. "Ill health sets the stage for allergy and aids reactions." Many of these female patients are neurotic.

The author analyses briefly the composition of powders, rouge, lipstick, creams, soaps, shampoos, shaving lotions, freckle removers, perfumes, hair restorers and dyes, eye shadow, eye drops, nail polishes and tooth paste. He outlines a scheme for the examination and diagnosis of such cases and gives a useful questionnaire; finally the treatment of the acute, subacute and chronic stages together with the general treatment. The latter section contains much shrewd advice: e.g., "keep your patients off the seven Cs: cocktails, coffee, condiments, cigarettes, carbo-hydrate, chocolate, and all cosmetics, of course, until you are sure of their innocence." With the increasing use of cosmetics it is not to be wondered at that such cases of circumocular dermatitis are on the increase. The ophthalmic surgeon who fails to recognize the cause of such a condition and treats the case with lotions and simple ointments as if it were some out of the way conjunctival or blepharitic condition is not likely to have much success and may easily have his "cosmetic eye" wiped by some more alert colleague if the patient does not rapidly improve. And it must be remembered that few of our female patients are sufficiently philosophically minded to put up with facial inflammation, obvious to the inspection of all their friends, without being in a great hurry to get it well. Dr. Ereaux's paper is quite short and well worth the attention of all eye surgeons. 